\title{
Extra abdominal Umbilical Vein Varix Causing Stillbirth: A Case Report
}

\author{
Luis Humberto Cruz Contreras ${ }^{1}$, Haneen Adnan Al-Maghrabi²*, Sareni Chávez Martínez ${ }^{1}$ \\ ${ }^{1}$ Department of Pathology, Mother and Child Hospital, Irapuato, Guanajuato, México \\ ${ }^{2}$ Department of Pathology, King Faisal Specialist Hospital and Research Center, Jeddah, Saudi Arabia
}

\begin{abstract}
Stillbirth continues to be one of the most devastating events for patients and doctors. Efforts for determining the cause of death should always be made, to prevent endangering future pregnancies and obstetric complications. A comprehensive approach that includes autopsy, placental examination and ancillary studies such as microbiology and toxicology are the best way of establishing the cause of stillbirth. Careful examination of the placenta and umbilical cord is imperative since they are associated with common causes of stillbirth. Here we present a case of umbilical vein varix that presented with associated complications that resulted in intrauterine fetal death.
\end{abstract}

Keywords: Stillbirth, Umbilical Vein Varix, Extra Abdominal

\section{Introduction}

The death of a formed fetus is one of the most emotionally devastating events for parents and clinicians. ${ }^{[1]}$ The estimated stillbirth rate worldwide is around 3.2 million per year with the vast majority of the cases occurring in developing countries. ${ }^{[2]}$ Fetal death that occurs before the stage of legal viability is called late spontaneous abortion or miscarriage while fetal death after the stage of legal viability is called intrauterine fetal death or stillbirth. ${ }^{[3]}$ The death is indicated by the fact that after separation from the mother the fetus does not breathe or show any other evidence of life, such as beating heart, pulsation of the umbilical cord, or definite movement of voluntary muscles. [3] Once fetal death has occurred delivery may be safely accomplished either medically or surgically and expectant management is a safe alternative for interested patients. It is often difficult to determine a "certain" cause of death. ${ }^{[1]}$ Common causes and risk factors for fetal death include chromosomal abnormalities, genetic syndromes, infections, placental abnormalities, fetal-maternal hemorrhage, maternal diseases such as diabetes and hypertension, antiphospholipid syndrome, thrombophilias, and abnormalities of multiple gestations. ${ }^{[1]}$ Over $50 \%$ of fetal deaths are related to placental causes. ${ }^{[4]}$ Autopsy, placental examination, and ancillary studies such as microbiology and toxicology are the best way of determining the cause of stillbirth. Efforts should be made at determining the cause since subsequent pregnancies may be at increased risk for fetal death and obstetric complications. ${ }^{[1]}$ Statistics and knowledge gained from post-mortem examinations are an essential part of clinical practice, which facilitates the research of stillbirth and is a crucial step towards its prevention. ${ }^{[3]}$

\section{Case Report}

We present the case of a 31-year-old women in her third pregnancy (Gravida 3, Para 1, abortus 1) without any relevant personal and family history, she had been attending prenatal care for low-risk pregnancies adequately, with a total of 6 visits to the clinic, all of them reported a pregnancy with normal evolution. She presented to the obstetric emergency department at 38 gestational weeks referring that she stopped perceiving fetal movements. Physical examination and ultrasound demonstrated intrauterine fetal death. The Patient underwent labor induction for vaginal delivery. Delivery was accomplished a day after she presented to our institution. A female stillbirth product was obtained. Amniotic fluid was referred as bloody by the attending gynecologist. The umbilical cord was cut and both Fetus and Placenta and were sent to the pathology department for post-mortem examination. The patient had no further complications.

In pathology department, we received a female term stillbirth with no external malformations. The umbilical cord had been previously sectioned from the placenta. The segment attached to the abdominal wall (9 cm length) had avariable diameter, was ulcerated and hemorrhagic (Fig1).

Serial sections of this segment revealed two normal arteries and an abnormal umbilical vein with irregular wall and a dilated lumen to a maximum of $1.9 \mathrm{~cm}$, with progressive thinning of the surrounding Wharton's Jelly and areas with ulceration. Additionally, an umbilical cord hematoma 
(1.9 $\mathrm{cm}$ greatest dimension) was found in close proximity to the dilated umbilical vein which was compressed. Placental findings were prominent vascular congestion

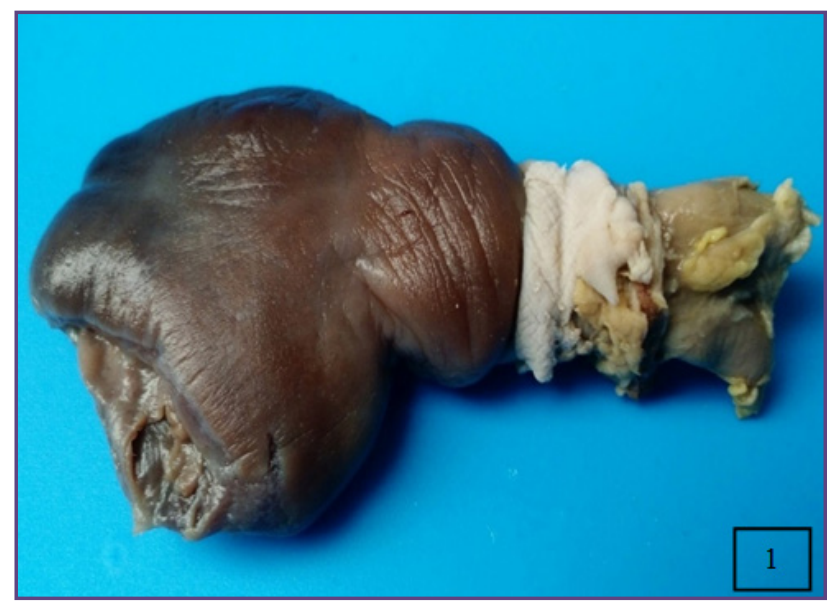

Fig. 1: Gross examination of the external aspect of umbilical cord (fetal insertion) which showsdilated and hemorrhagic outer surface. and focal hemorrhage (Fig. 2 and 3). The rest of the post mortem examination revealed only generalized vascular congestion. No other abnormalities were found.

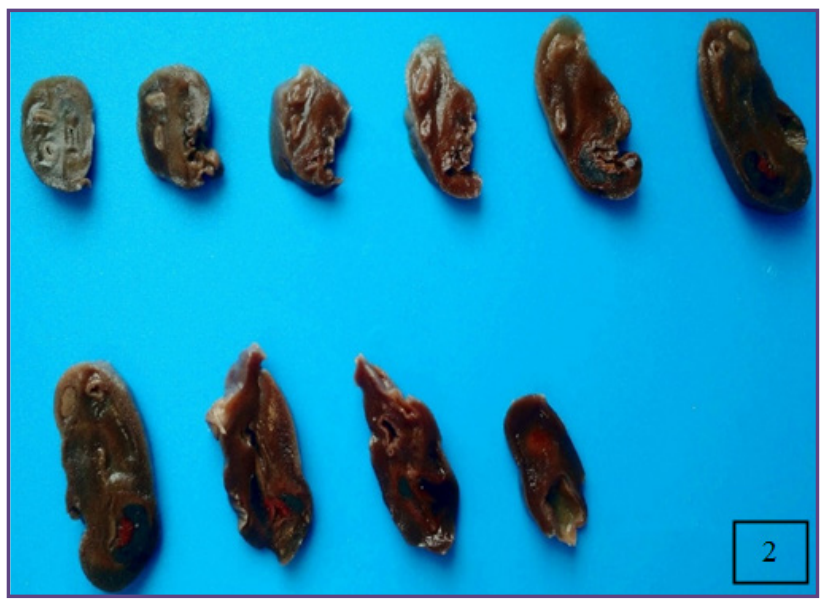

Fig. 2: Serial sections of the cord showing progressive dilatation of the umbilical vein (left to right, top to bottom) with progressive thinning of surrounding Wharton Jelly with ulceration and associated cord hematoma.

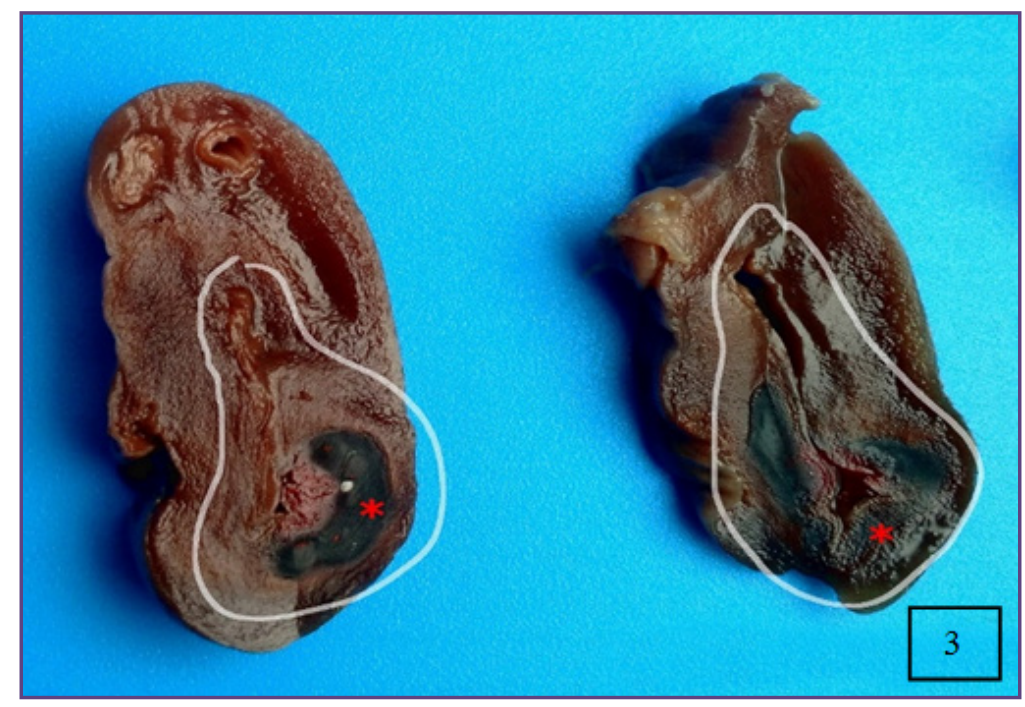

Fig. 3: Two cord sections (close-up) showing in detail the umbilical vein dilatation and cord hematoma $(*)$.

\section{Discussion}

The umbilical cord enables the fetus to develop, grow, adapt, survive andborn healthy. Some umbilical cords abnormalities are well-established factors for gestational complications and impairment of fetal wellbeing. [5] Umbilical vein varix , a localized dilatation of vein diameter can present either intra-abdominal or extraabdominal (intra-amniotic). ${ }^{[6]}$ Beraud and his colleagues defined the intra-abdominal umbilical vein varix according to two criteria; umbilical vein diameter greater than or equal to $9 \mathrm{~mm}$, and an increase in the subhepatic segment diameter greater than $50 \%$ of the intrahepatic segment diameter. ${ }^{[7]}$ This focal intra-abdominal vein dilatation is diagnosed by ultrasonography as dilated segment at the axial section of the abdomen. Thus, it is essential to examine the intra-abdominal portion of the umbilical vein. It is believed that the intra-abdominal segment can be examined and visualized easily in comparison with 
the extra-abdominal vein varix. Ultrasound examination of the extra-abdominal vein varix usually shows normal umbilical vein study with no increase in diameter. Some cases might lead to hematoma and surface ulceration like our case. These changes might lead to bradycardia and caesarean section should be considered. As Fung and his group found in their studies that intra-abdominal varix of the umbilical vein implies a higher incidence of adverse perinatal outcomes, especially the fetal complications like anemia, chromosomal abnormalities, and intrauterine fetal death ${ }^{[8]}$ Cardiovascular anomalies can be seen frequently associated with trisomy 21 and 18. Other reported anomalies are trisomy 9, triploidy and mosaics like Turner syndrome. ${ }^{[8]}$ Thus, careful ultrasound cardiac examination is required. Most of the literature had reviewed intraabdominal umbilical vein dilatation, but only a few studies report cases of extra-abdominal umbilical vein varix. ${ }^{[6]}$ Due to the extreme difficulties in diagnosis at the prenatal period, it is usually made as post-natal diagnosis and confirmed by histopathology exam. Sadly, the majority of cases were diagnosed retrospectively during autopsy of antepartum dead fetus. ${ }^{[9]}$ Moreover, there is an increase in the risk of stillbirth, fetal heart failure, thrombosis, and hydrops fetalis. ${ }^{[8,10]}$ Kanenishi and his colleagues report 13 cases of extra-abdominal intra-amniotic umbilical vein varices, in which half of them were diagnosed after birth. ${ }^{[11]}$ This shows how difficult it is to diagnose it before birth. Intra-amniotic umbilical vein varix is associated with high frequency of umbilical vein thrombosis, antepartum fetal death, fetal anomalies and neonatal coagulation problems. ${ }^{[11]}$ In these cases, the rate of caesarean sections is elevated with a good fetal outcome. Most of the studies in the literature emphasis the importance of prenatal diagnosis and regular follow up in order to establish the appropriate treatment, once the fetal lung is mature. However, the diagnosis of cases with extra-abdominal intra-amniotic umbilical vein varices remains a big diagnostic challenge by ultrasound studies. There are some risk factors that can lead to umbilical cord mechanical compression and blood ectasias, these include umbilical cord length, long cord (more than $70 \mathrm{~cm}$ ) or short cord (less than $35 \mathrm{~cm}$ ), reduced diameter (less than $8.0 \mathrm{~mm}$ ), and excessive cord twisting (more than $0.3 \mathrm{~cm} / \mathrm{loop}$ ). ${ }^{[5]}$ These might lead to serious complications like arterial or venous thrombosis, hematoma and ulceration. ${ }^{[5,12,13]}$ Ulceration of the umbilical cord is one of the complications that can result in alteration of fetal heart rate. Many theories had been developed to explain the cases of umbilical cord ulceration. These include vascular theory, reflux theory, and epithelial abnormality. ${ }^{[13]}$ The first theory is related to vascular changes and ischemic changes which will lead to surface ulceration. The second theory is related to chemical material reflux like gastric and intestinal digestive enzymes effects. And the third theory is related to the abnormal epithelial changes like the bulla formation. ${ }^{[10]}$ Other related causes of umbilical cord ulceration include smoking, patent omphalomesenteric duct, absence of Wharton's jelly and injury by fetal nail. ${ }^{[13]}$

\section{Conclusion}

Extra-abdominal intra-amniotic umbilical vein varix is an uncommon anomaly. There are numerous reports in the literature about intra-abdominal vein varix which can be easily detected by ultrasound examination. Sadly, most of the extra-abdominal vein varix are detected in the post-natal period. Delivery of the fetus, as soon as lung maturation is confirmed, is recommended to reduce umbilical cord complication and fetal distress.

\section{References}

1. Silver RM., et al., Work-up of stillbirth: a review of the evidence. American journal of obstetrics and gynecology, 2007. 196(5): 433-444.

2. Abraham A., et al., Umbilical Cord Haematoma Causing Still Birth-A Case Report. Journal of clinical and diagnostic research: JCDR, 2015. 9(12): QD01.

3. Lehtonen, T., et al., Causes of Stillbirth in Turku, Finland, 2001-2011. Pediatric and Developmental Pathology, 2017. 20(1):5-15.

4. Boyd, T.K., et al., The Stillbirth Classification System for the Safe Passage Study: Incorporating Mechanism, Etiology, and Recurrence. Pediatric and Developmental Pathology, 2016: p. 1093526616686251.

5. Oliveira, G.H.d., et al., Intrauterine thrombosis of umbilical artery-case report. Sao Paulo Medical Journal, 2016 (AHEAD).

6. Crespo, V., Variz venosa umbilical intramniótica. Reporte de un caso. Ginecol Obstet Mex, 2015. 83:356-362.

7. Beraud, E., et al., Umbilical vein varix: Importance of anteand post-natal monitoring by ultrasound. Diagnostic and interventional imaging, 2015. 96(1): 21-26.

8. Fung, T., et al., Fetal intra $\square$ abdominal umbilical vein varix: what is the clinical significance? Ultrasound in obstetrics \& gynecology, 2005. 25(2): 149-154.

9. Cruise, K.R. and G. Rouse, Klippel-Trenaunay-Weber syndrome complicated by extrafetal umbilical vein varix. Journal of Diagnostic Medical Sonography, 2002. 18(5):317-320. 
10. Sepulveda, W., et al., Fetal prognosis in varix of the intrafetal umbilical vein. Journal of ultrasound in medicine, 1998. 17(3): 171-175.

11. Dussaux, C., et al., Umbilical vein thrombosis: to deliver or not to deliver at the time of diagnosis? Clinical case reports, 2014. 2(6):271-273
12. Viora, E., et al., Thrombosis of umbilical vein varix. Ultrasound in Obstetrics and Gynecology, 2002. 19(2): 212-213.

13. Maheshwari, B., et al., Umbilical cord ulceration: An underdiagnosed entity. Obstetrics \& Gynecology Science, 2016. 59(5):388-392.

*Corresponding author:

Haneen Adnan Al-Maghrabi, Department of Pathology, King Faisal Specialist Hospital and Research Center, Jeddah, Saudi Arabia Email: haneen.almaghrabi@hotmail.com

Date of Submission : 07.01.2017

Date of Acceptance : 24.02.2017

Financial or other Competing Interests: None.

Date of Publication : 29.05.2017 